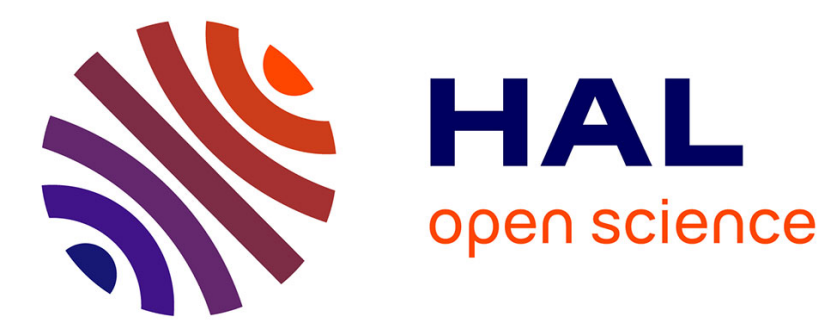

\title{
Topological fixed points in Boolean networks
}

\author{
Loïc Paulevé, Adrien Richard
}

\section{To cite this version:}

Loïc Paulevé, Adrien Richard. Topological fixed points in Boolean networks. Comptes rendus de l'Académie des sciences. Série I, Mathématique, 2010, 348 (15-16), pp.825-828. 10.1016/j.crma.2010.07.014 . hal-00510892

\section{HAL Id: hal-00510892 \\ https://hal.science/hal-00510892}

Submitted on 16 Jun 2016

HAL is a multi-disciplinary open access archive for the deposit and dissemination of scientific research documents, whether they are published or not. The documents may come from teaching and research institutions in France or abroad, or from public or private research centers.
L'archive ouverte pluridisciplinaire HAL, est destinée au dépôt et à la diffusion de documents scientifiques de niveau recherche, publiés ou non, émanant des établissements d'enseignement et de recherche français ou étrangers, des laboratoires publics ou privés. 


\title{
Topological Fixed Points in Boolean Networks
}

\author{
Loïc Paulevéa ${ }^{\mathrm{a}}$ Adrien Richard ${ }^{\mathrm{b}}$ \\ ${ }^{a}$ IRCCyN, École centrale de Nantes, 1 rue de la Noë, 44321 Nantes, France. \\ ${ }^{b}$ I3S, UMR 6070 CNRS E Université de Nice-Sophia Antipolis, \\ 2000 route des Lucioles, 06903 Sophia Antipolis, France.
}

\begin{abstract}
We introduce the notion of a topological fixed point in Boolean Networks: a fixed point of Boolean network $F$ is said topologic if it is a fixed point of every Boolean network with the same interaction graph as the one of $F$. Then, we characterize the number of topological fixed points of a Boolean network according to the structure of its interaction graph.
\end{abstract}

\section{Résumé}

Points fixes topologiques dans les réseaux booléens. Nous introduisons la notion de point fixe topologique dans les réseaux booléens : un point fixe d'un réseau booléen $F$ est dit topologique s'il est un point fixe de tous les réseaux booléens ayant le même graphe d'interaction que $F$. Ensuite, nous caractérisons le nombre de points fixes topologiques d'un réseau booléen en fonction de la structure de son graphe d'interaction.

\section{Introduction}

We are interested by the relationships between the stable states and the topology of Boolean networks. On one side, the dynamics of a Boolean network with $n$ components is usually described by the successive iterations of a map $F$ from $\{0,1\}^{n}$ to itself. The stable states of the network then correspond to the fixed points of $F$. On the other side, the topology of the network is often described by an interaction graph $G$ that can be deduced from $F$. The vertices correspond to the network components, and the edges, which are directed and signed, describe causal relationships in terms of activations and inhibitions between components.

Boolean networks have been applied in many area, especially for modeling gene networks (see, for instance, the work of Kauffman [4, 5] and Thomas [7, 8]). The relationships between $G$ and the fixed points of $F$ are of particular interest in this context: fixed points have often biological meanings (e.g. stable patterns of gene expressions corresponding to particular cellular functions) $[5,8,6]$, and the first reliable informations obtained when biologists study gene networks are often represented in terms of interaction graphs [3].

In this note, we focus on the fixed points of $F$ that only depend on $G$, and we says that these are the topological fixed points of $F$. Topological fixed points of $F$ can be seen as "robust" fixed points in the sense that they remain fixed points after any perturbation of $F$ that does affect the interaction graph $G$ of the network. As main result, we characterize the number of topological fixed points of $F$ according to the structure of $G$. This characterization uses and generalizes a theorem of Aracena, Demongeot and Goles [1,2].

\section{Definitions}

Interaction graph. An $n$-interaction graph $\mathscr{G}$ is a directed graph on $\{1, \ldots, n\}$ in which each arc $j i$ (from $j$ to $i$ ) is either positive, negative or unsigned. The set of positive, negative and unsigned $\operatorname{arcs}$ of $\mathscr{G}$ is denoted by $\mathscr{G}^{+}, \mathscr{G}^{-}$, and

Email addresses: loic.pauleve@irccyn.ec-nantes.fr (Loïc Paulevé), richard@i3s.unice.fr (Adrien Richard) 
$\mathscr{G}^{0}$, respectively. The set of positive (resp. negative, unsigned) predecessors of a vertex $i$ is $\mathscr{G}_{i}^{+}=\left\{j \mid j i \in \mathscr{G}^{+}\right\}$(resp. $\left.\mathscr{G}_{i}^{-}=\left\{j \mid j i \in \mathscr{G}^{-}\right\}, \mathscr{G}_{i}^{0}=\left\{j \mid j i \in \mathscr{G}^{0}\right\}\right)$. The set of signed predecessors of $i$ is $\mathscr{G}_{i}^{+} \cup \mathscr{G}_{i}^{-}$. An undirected path of $\mathscr{G}$ is a sequence of $p \geq 1$ vertices $i_{0} i_{1} \ldots i_{p}$ such that $i_{k} i_{k+1}$ or $i_{k+1} i_{k}$ is an arc of $\mathscr{G}, 0 \leq k<p$. Such a path joins $i_{0}$ and $i_{p}$, and is a cycle if $i_{0}=i_{p}$. An undirected path without unsigned arc is signed. A signed undirected path is positive if it contains an even number of negative arcs, and negative otherwise. $\mathscr{G}$ is connected if there exists an undirected path joining each pair of distinct vertices. A connected component of $\mathscr{G}$ is a maximal subset $C$ of vertices with the property that $\mathscr{G}$ has an undirected path joining each pair of distinct vertices taken in $C$. We denote by $\tilde{\mathscr{G}}$ the $n$-interaction graph that we obtain by removing the unsigned $\operatorname{arcs}$ of $\mathscr{G}$. Formally, $\tilde{\mathscr{G}}$ is the $n$-interaction graph such that $\tilde{\mathscr{G}}^{+}=\mathscr{G}^{+}$, $\tilde{\mathscr{G}}^{-}=\mathscr{G}^{-}$and $\tilde{\mathscr{G}}^{0}=\emptyset$.

Topological fixed point. Consider a Boolean map

$$
F=\left(f_{1}, \ldots, f_{n}\right):\{0,1\}^{n} \rightarrow\{0,1\}^{n}, \quad x=\left(x_{1}, \ldots, x_{n}\right) \mapsto F(x)=\left(f_{1}(x), \ldots, f_{n}(x)\right) .
$$

The discrete derivative of $f_{i}$ with respect to the variable $x_{j}$ is the map $f_{i j}:\{0,1\}^{n} \rightarrow\{-1,0,1\}$ defined by:

$$
f_{i j}(x)=f_{i}\left(x_{1}, \ldots, x_{j-1}, 1, x_{j+1}, \ldots, x_{n}\right)-f_{i}\left(x_{1}, \ldots, x_{j-1}, 0, x_{j+1}, \ldots, x_{n}\right) \quad(i, j=1, \ldots, n) .
$$

The interaction graph of $F$ is the $n$-interaction graph $G(F)$ defined by: for $i, j=1, \ldots, n$, there exists an arc $j i$ if $f_{i j} \neq 0$, and this arc is positive if $f_{i j} \geq 0$, negative if $f_{i j} \leq 0$, and unsigned otherwise (that is, if $f_{i j}$ is somewhere positive and somewhere negative). Note that $f_{i j} \neq 0$ if and only if the value of $f_{i}$ depends on the value of $x_{j}$. A point $x \in\{0,1\}^{n}$ is a fixed point of $F$ if $F(x)=x$, and $x$ is a topological fixed point of $F$ if it is a fixed point of every map $H:\{0,1\}^{n} \rightarrow\{0,1\}^{n}$ such that $G(H)=G(F)$.

Admissible interaction graph. We say that an $n$-interaction graph $\mathscr{G}$ is admissible if there exists $F:\{0,1\}^{n} \rightarrow\{0,1\}^{n}$ such that $G(F)=\mathscr{G}$. If $\mathscr{G}$ is admissible, we say that $x \in\{0,1\}^{n}$ is a topological fixed point of $\mathscr{G}$ if $x$ is a fixed point of every map $F:\{0,1\}^{n} \rightarrow\{0,1\}^{n}$ such that $G(F)=\mathscr{G}$. So $x$ is a topological fixed point of $F$ if and only if $x$ is a topological fixed point of $G(F)$.

Boolean operations. We set $\overline{0}=\sigma^{-}(0)=\sigma^{+}(1)=1 ; \overline{1}=\sigma^{-}(1)=\sigma^{+}(0)=0$; and $\bar{x}=\left(\overline{x_{1}}, \overline{x_{2}}, \ldots, \overline{x_{n}}\right)$. A sum of Boolean variables is always a Boolean sum $(1+1=1)$, and the sum modulo 2 is denoted by $\oplus$. By convention, the empty product is 1 and the empty sum is 0 .

\section{Results}

Let $F$ be a map from $\{0,1\}^{n}$ to itself such that $G(F)$ has no unsigned arc. We say that $G(F)$ has the property $\mathcal{P}$ if $G(F)$ is connected, if each vertex of $G(F)$ has a predecessor, and if $G(F)$ has no undirected negative cycle. Aracena, Demongeot and Goles $[2,1]$ proved a theorem that can be stated as follows with our notations:

If $G(F)$ has the property $\mathcal{P}$, then there exists $x \in\{0,1\}^{n}$ such that $x$ and $\bar{x}$ are fixed points of $F$.

An easy unmentioned consequence of their constructive proof (that we use and extend here) is that $x$ and $\bar{x}$ are actually topological fixed points of $F$, and that no other topological fixed point exists. So, given an $n$-interaction graph $\mathscr{G}$ without unsigned arcs (such a graph is always admissible, cf. Remark 4.2 below), we have the following:

If $\mathscr{G}$ has the property $\mathcal{P}$, then $\mathscr{G}$ has exactly two topological fixed points, and these are of the form $x, \bar{x}$.

In the following, we show that the converse of this slightly stronger version of the theorem of Aracena et al is true:

If $\mathscr{G}$ has exactly two topological fixed points, then these are of the form $x, \bar{x}$, and $\mathscr{G}$ has the property $\mathcal{P}$.

The two above statements are in fact contained in the following theorem, which provides a characterization of the number of topological fixed points of any admissible interaction graph:

Theorem 3.1. Let $\mathscr{G}$ be an admissible n-interaction graph.

(1) Let $p$ be the number of connected components of $\tilde{\mathscr{G}}$. If each vertex of $\mathscr{G}$ has a predecessor and at most one unsigned predecessor, and if $\mathscr{G}$ has no undirected negative cycle, then $\mathscr{G}$ has exactly $2^{p}$ topological fixed points. Otherwise, $\mathscr{G}$ has 0 topological fixed point.

(2) If $x$ is a topological fixed point of $\mathscr{G}$, then $\bar{x}$ is also a topological fixed point of $\mathscr{G}$. 
Remark 3.2. If $\mathscr{G}$ has $m$ arcs, then the number of connected components of $\tilde{\mathscr{G}}$ can be computed in $O(n+m)$, and the presence of an undirected negative cycle in $\mathscr{G}$ can be checked with the same complexity. So following Theorem 3.1, the number of topological fixed points of $\mathscr{G}$ can be computed in $O(n+m)$.

\section{Proof of Theorem 3.1}

We begin with a basic lemma on unsigned arcs.

Lemma 4.1. Let $\mathscr{G}$ be an $n$-interaction graph. If $\mathscr{G}$ is admissible, then every vertex of $\mathscr{G}$ with a unique unsigned predecessor has at least two signed predecessors.

Proof - Suppose that $\mathscr{G}$ is admissible, and that $\mathscr{G}$ has a vertex $i$ with a unique unsigned predecessor, say $k$. Then, for each $F:\{0,1\}^{n} \rightarrow\{0,1\}^{n}$ such that $G(F)=\mathscr{G}$, there exists $x, y \in\{0,1\}^{n}$ such that:

$$
\begin{aligned}
& 0=f_{i}\left(x_{1}, \ldots, x_{k-1}, 0, x_{k+1}, \ldots, x_{n}\right)<f_{i}\left(x_{1}, \ldots, x_{k-1}, 1, x_{k+1}, \ldots, x_{n}\right)=1, \\
& 1=f_{i}\left(y_{1}, \ldots, y_{k-1}, 0, y_{k+1}, \ldots, y_{n}\right)>f_{i}\left(y_{1}, \ldots, y_{k-1}, 1, y_{k+1}, \ldots, y_{n}\right)=0 .
\end{aligned}
$$

So the value of $f_{i}$ depends on the value of at least one variable $x_{j}, j \neq k$, and it is easy to see that if $f_{i}$ only depends on $x_{k}$ and $x_{j}$, then $j$ is another unsigned predecessor of $i$, a contradiction.

Remark 4.2. This necessary condition for admissibility is also sufficient. (Indeed, if each vertex of $\mathscr{G}$ with a unique unsigned predecessor has at least two signed predecessors, then it is easy to see that $\mathscr{G}$ is the interaction graph of the map $F$ defined by: (1) for each $i$ without unsigned predecessor, $f_{i}(x)=\sum_{j \in G_{i}^{+}} x_{j}+\sum_{j \in G_{i}^{-}} \overline{x_{j}}$; (2) for each $i$ with a unique unsigned predecessor $k, f_{i}(x)=x_{k} \sigma^{s_{1}}\left(x_{l_{1}}\right)+\overline{x_{k}} \sigma^{s_{2}}\left(x_{l_{2}}\right)+\sum_{j \in G_{i}^{+} \backslash\left\{l_{1}, l_{2}\right\}}+\sum_{j \in G_{i}^{-} \backslash\left\{l_{1}, l_{2}\right\}} \overline{x_{j}}$, where $l_{1} \in \mathscr{G}_{i}^{s_{1}}$ and $l_{2} \in \mathscr{G}_{i}^{s_{2}}$ are two signed predecessors of $i$; (3) for each $i$ with $p \geq 2$ unsigned predecessors $k_{1}, \ldots, k_{p}, f_{i}(x)=$ $\sum_{1 \leq q<p}\left(x_{k_{q}} \oplus x_{k_{q+1}}\right)+\sum_{j \in \mathscr{G}_{i}^{+}} x_{j}+\sum_{j \in \mathscr{G}_{i}^{-}} \overline{x_{j}}$.

The main lemma follows.

Lemma 4.3. Let $\mathscr{G}$ be an admissible $n$-interaction graph. A point $\alpha \in\{0,1\}^{n}$ is a topological fixed point of $\mathscr{G}$ if and only if (1) every vertex of $\mathscr{G}$ has a predecessor and at most one unsigned predecessor, and (2) $\alpha_{j}=\alpha_{i}$ for all ji $\in \mathscr{G}^{+}$, and $\alpha_{j} \neq \alpha_{i}$ for all $j i \in \mathscr{G}^{-}$.

Proof - (Sufficient condition) Let $F:\{0,1\}^{n} \rightarrow\{0,1\}^{n}$ be such that $G(F)=\mathscr{G}$, and let us show that $\alpha$ is a fixed point of $F$. Suppose, by contradiction, that there exists a vertex $i$ such that $f_{i}(\alpha) \neq \alpha_{i}$. If $i$ has no unsigned predecessor, we set $X=\left\{x \mid f_{i}(x)=\alpha_{i}\right\}$; since $i$ has a predecessor, $f_{i}$ is not constant, so $X$ is not empty. If $i$ has a unique unsigned predecessor, say $k$, we set $X=\left\{x \mid f_{i}(x)=\alpha_{i}, x_{k}=\alpha_{k}\right\}$; since $k$ is an unsigned predecessor of $i$, for all $a, b \in\{0,1\}$, there exists $x$ such that $f_{i}(x)=a$ and $x_{k}=b$, so $X$ is not empty. Let $x$ be a point of $X$ minimizing the Hamming distance $d(x, \alpha)$, that is, the number of $j \in\{1, \ldots, n\}$ such that $x_{j} \neq \alpha_{j}$. Since $f_{i}(x) \neq f_{i}(\alpha)$, there exists $j$ such that $x_{j} \neq \alpha_{j}$, and, by construction, $j$ is not an unsigned predecessor of $i$. Consider the point $y$ such that $y_{j}=\overline{x_{j}}=\alpha_{j}$ and $y_{l}=x_{l}$ for every vertex $l \neq j$. We have $d(y, \alpha)=d(x, \alpha)-1$. So $y \notin X$, and we deduce that $f_{i}(y) \neq f_{i}(x)=\alpha_{i}$. So $f_{i j}(x)>0$ if $\alpha_{i}=x_{j}$, and $f_{i j}(x)<0$ if $\alpha_{i} \neq x_{j}$. Since $j$ is not an unsigned predecessor of $i$, and since $x_{j} \neq \alpha_{j}$, we deduce that either $j i \in \mathscr{G}^{+}$and $\alpha_{i} \neq \alpha_{j}$, or $j i \in \mathscr{G}^{-}$and $\alpha_{i}=\alpha_{j}$, a contradiction. So $f_{i}(\alpha)=\alpha_{i}$ for all vertex $i$.

(Necessary condition) Suppose that $\alpha$ is a topological fixed point of $\mathscr{G}$. We will show that conditions (1) and (2) hold for an arbitrary given vertex $i$. Let us say that $h_{i}:\{0,1\}^{n} \rightarrow\{0,1\}$ is admissible if there exists $F:\{0,1\}^{n} \rightarrow\{0,1\}^{n}$ such that $f_{i}=h_{i}$ and $G(F)=\mathscr{G}$. So if $h_{i}$ is admissible, then $h_{i}(\alpha)=\alpha_{i}$.

Suppose that $i$ has $p \geq 2$ unsigned predecessors $k_{1}, \ldots, k_{p}$. Consider the four following maps from $\{0,1\}^{n}$ to $\{0,1\}$ :

$$
\begin{array}{ll}
h_{i}^{1}(x)=\prod_{1 \leq q<p}\left(x_{k_{q}} \oplus x_{k_{q+1}}\right) \cdot \prod_{j \in \mathscr{G}_{i}^{+}} x_{j} \cdot \prod_{j \in \mathscr{G}_{i}^{-}} \overline{x_{j},} & h_{i}^{3}(x)=\sum_{1 \leq q<p}\left(x_{k_{q}} \oplus x_{k_{q+1}}\right)+\sum_{j \in \mathscr{G}_{i}^{+}} x_{j}+\sum_{j \in \mathscr{G}_{i}^{-}} \overline{x_{j}}, \\
h_{i}^{2}(x)=\prod_{1 \leq q<p}\left(\overline{x_{k_{q}} \oplus x_{k_{q+1}}}\right) \cdot \prod_{j \in \mathscr{G}_{i}^{+}} x_{j} \cdot \prod_{j \in \mathscr{G}_{i}^{-}} \overline{x_{j},} & h_{i}^{4}(x)=\sum_{1 \leq q<p}\left(\overline{x_{k_{q}} \oplus x_{k_{q+1}}}\right)+\sum_{j \in \mathscr{G}_{i}^{+}} x_{j}+\sum_{j \in \mathscr{G}_{i}^{-}} \overline{x_{j} .}
\end{array}
$$

It is easy to see that $h_{i}^{r}$ is admissible for $r=1,2,3,4$. So $h_{i}^{r}(\alpha)=\alpha_{i}$ for $r=1,2,3,4$. But if $h_{i}^{1}(\alpha)=1$ then $h_{i}^{2}(\alpha)=0$, and if $h_{i}^{3}(\alpha)=0$ then $h_{i}^{4}(\alpha)=1$. We deduce that $h_{i}^{1}(\alpha) \neq h_{i}^{2}(\alpha)$ or $h_{i}^{3}(\alpha) \neq h_{i}^{4}(\alpha)$, a contradiction. So $i$ has at most one unsigned predecessor. So we have the two following cases. 
Case 1: the vertex $i$ has no unsigned predecessor. Consider the two following maps from $\{0,1\}^{n}$ to $\{0,1\}$ :

$$
h_{i}^{1}(x)=\prod_{j \in \mathscr{G}_{i}^{+}} x_{j} \cdot \prod_{j \in \mathscr{G}_{i}^{-}} \overline{x_{j}}, \quad h_{i}^{2}(x)=\sum_{j \in \mathscr{G}_{i}^{+}} x_{j}+\sum_{j \in \mathscr{G}_{i}^{-}} \overline{x_{j}} .
$$

It is easy to see that $h_{i}^{1}$ and $h_{i}^{2}$ are admissible, so $h_{i}^{1}(\alpha)=h_{i}^{2}(\alpha)=\alpha_{i}$. If $i$ has no predecessor, then $h_{i}^{1}(\alpha)=1$ and $h_{i}^{2}(\alpha)=0$, a contradiction. So $i$ has a predecessor, and condition (1) holds for $i$. We now prove that the condition (2) holds too. Suppose that $j i \in \mathscr{G}^{+}$. If $\alpha_{i}=1$ then $h_{i}^{1}(\alpha)=1$ thus $\alpha_{j}=1$, and if $\alpha_{i}=0$ then $h_{i}^{2}(\alpha)=0$ thus $\alpha_{j}=0$. So in both cases, $\alpha_{j}=\alpha_{i}$. We prove similarly that $\alpha_{j} \neq \alpha_{i}$ for every $j i \in \mathscr{G}^{-}$.

Case 2: the vertex $i$ has a unique unsigned predecessor $k$. It is sufficient to prove that condition (2) holds. By Lemma 4.1, $i$ has at least two signed predecessors, say $l_{1} \in \mathscr{G}_{i}^{s_{1}}$ and $l_{2} \in \mathscr{G}_{i}^{s_{2}}$. Consider the four following maps:

$$
\begin{aligned}
& h_{i}^{1}(x)=x_{k} \sigma^{s_{1}}\left(x_{l_{1}}\right)+\overline{x_{k}} \sigma^{s_{2}}\left(x_{l_{2}}\right)+\sum_{j \in \mathscr{G}_{i}^{+} \backslash\left\{l_{1}, l_{2}\right\}} x_{j}+\sum_{j \in \mathscr{G}_{i}^{-} \backslash\left\{l_{1}, l_{2}\right\}} \overline{x_{j}}, \\
& h_{i}^{2}(x)=x_{k} \sigma^{s_{2}}\left(x_{l_{2}}\right)+\overline{x_{k}} \sigma^{s_{1}}\left(x_{l_{1}}\right)+\sum_{j \in \mathscr{G}_{i}^{+} \backslash\left\{l_{1}, l_{2}\right\}} x_{j}+\sum_{j \in \mathscr{G}_{i}^{-} \backslash\left\{l_{1}, l_{2}\right\}} \overline{x_{j}}, \\
& h_{i}^{3}(x)=\left(x_{k}+\sigma^{s_{1}}\left(x_{l_{1}}\right)\right)\left(\overline{x_{k}}+\sigma^{s_{2}}\left(x_{l_{2}}\right)\right) \prod_{j \in \mathscr{G}_{i}^{+} \backslash\left\{l_{1}, l_{2}\right\}} x_{j} \prod_{j \in \mathscr{G}_{i}^{-} \backslash\left\{l_{1}, l_{2}\right\}} \overline{x_{j}}, \\
& h_{i}^{4}(x)=\left(x_{k}+\sigma^{s_{2}}\left(x_{l_{2}}\right)\right)\left(\overline{x_{k}}+\sigma^{s_{1}}\left(x_{l_{1}}\right)\right) \prod_{j \in \mathscr{G}_{i}^{+} \backslash\left\{l_{1}, l_{2}\right\}} x_{j} \prod_{j \in \mathscr{G}_{i}^{-} \backslash\left\{l_{1}, l_{2}\right\}} \overline{x_{j}} .
\end{aligned}
$$

It is easy to see that $h_{i}^{r}$ is admissible for $r=1,2,3,4$. So $h_{i}^{r}(\alpha)=\alpha_{i}$ for $r=1,2,3,4$. For all $j \in\left(\mathscr{G}_{i}^{+} \cup \mathscr{G}_{i}^{-}\right) \backslash\left\{l_{1}, l_{2}\right\}$, we prove, as in the first case, that $\alpha_{j}=\alpha_{i}$ if $j i \in \mathscr{G}^{+}$and $\alpha_{j} \neq \alpha_{i}$ if $j i \in \mathscr{G}^{-}$. Then, if $\alpha_{i}=0$ we have $h_{i}^{1}(\alpha)=h_{i}^{2}(\alpha)=0$, and we deduce that $\sigma^{s_{1}}\left(\alpha_{l_{1}}\right)=\sigma^{s_{2}}\left(\alpha_{l_{2}}\right)=0$. If $\alpha_{i}=1$ then $h_{i}^{3}(\alpha)=h_{i}^{4}(\alpha)=1$, and we deduce that $\sigma^{s_{1}}\left(\alpha_{l_{1}}\right)=\sigma^{s_{2}}\left(\alpha_{l_{2}}\right)=1$. So $\sigma^{s_{1}}\left(\alpha_{l_{1}}\right)=\sigma^{s_{2}}\left(\alpha_{l_{2}}\right)=\alpha_{i}$ in both cases, and so the condition (2) holds for all the signed predecessors of $i$.

Remark 4.4. The condition (2) is equivalent to the condition "every undirected path of $\tilde{\mathscr{G}}$ joining $j$ and $i$ is positive if $\alpha_{j}=\alpha_{i}$, and negative if $\alpha_{j} \neq \alpha_{i}$ ”. As a consequence, if $\mathscr{G}$ has a topological fixed point, then $\tilde{\mathscr{G}}$ and $\mathscr{G}$ have no undirected negative cycle.

Remark 4.5. We deduce from Lemma 4.3 that if each vertex of $\mathscr{G}$ has at most one unsigned predecessor, then $\alpha$ is a topological fixed point of $\mathscr{G}$ if and only if $\alpha$ is a topological fixed point of $\tilde{\mathscr{G}}$.

The second part of Theorem 3.1 is an immediate consequence of Lemma 4.3. To prove the first part, we need a last lemma.

Lemma 4.6. Let $\mathscr{G}$ be an admissible n-interaction graph. If $\tilde{\mathscr{G}}$ is connected, if each vertex of $\mathscr{G}$ has a predecessor and at most one unsigned predecessor, and if $\mathscr{G}$ has no undirected negative cycle, then $\mathscr{G}$ has exactly 2 topological fixed points.

Proof - For each vertex $i \neq 1$, let $P_{1 i}$ be an undirected path of $\tilde{\mathscr{G}}$ joining 1 and $i$ ( $\tilde{\mathscr{G}}$ is connected). Let $\alpha \in\{0,1\}^{n}$ be defined by: $\alpha_{1}=0, \alpha_{i}=0$ if $P_{1 i}$ is positive, and $\alpha_{i}=1$ otherwise $(2 \leq i \leq n)$. If $j i \in \mathscr{G}^{+}$and $\alpha_{j} \neq \alpha_{i}$, then, by definition, $P_{1 j}$ and $P_{1 i}$ have opposite signs. So these paths, together with the positive arc $j i$, form an undirected negative cycle, a contradiction. We prove similarly that if $j i \in \mathscr{G}^{-}$then $\alpha_{j} \neq \alpha_{i}$. Consequently, according to Lemma 4.3, $\alpha$ and $\bar{\alpha}$ are topological fixed points of $\mathscr{G}$. Consider a point $\beta \neq \alpha, \bar{\alpha}$. Then there exists $i, j$ such that $\beta_{i}=\alpha_{i}$ and $\beta_{j} \neq \alpha_{j}$. Let $P$ be an undirected path of $\tilde{\mathscr{G}}$ joining $j$ and $i$ ( $\tilde{\mathscr{G}}$ is connected). According to Remark 4.4, $P$ is positive if and only if $\alpha_{j}=\alpha_{i}$. So $P$ is positive if and only if $\beta_{j} \neq \beta_{i}$, and according to the same remark, $\beta$ is not a topological fixed point of $\mathscr{G}$.

Suppose that $\tilde{\mathscr{G}}$ has $p$ connected components, and suppose that $\mathscr{G}$ has the following property $\mathcal{P}^{\prime}$ : each vertex of $\mathscr{G}$ has a predecessor and at most one unsigned predecessor, and $\mathscr{G}$ has no undirected negative cycle. Then, each connected component of $\tilde{\mathscr{G}}$ induces an interaction graph that satisfies the conditions of the previous lemma, and that has thus exactly two topological fixed points. It is then clear that $\tilde{\mathscr{G}}$ has exactly $2^{p}$ topological fixed points, and we deduce from Remark 4.5 that $\mathscr{G}$ has also $2^{p}$ topological fixed points. If $\mathscr{G}$ does not satisfy the property $\mathcal{P}^{\prime}$, following Lemma 4.3 and Remark 4.4, $\mathscr{G}$ has 0 topological fixed point. This completes the proof of Theorem 3.1.

[1] J. Aracena, Maximum number of fixed points in regulatory boolean networks, Bulletin of Mathematical Biology, 70 (2008) 1398-1409. 
[2] J. Aracena, J. Demongeot, E. Goles, Positive and negative circuits in discrete neural networks, IEEE Transactions of Neural Networks, 15 (2004) 77-83.

[3] H. de Jong, Modeling and Simulation of Genetic Regulatory Systems: A Literature Review, Journal of Computational Biology, 9 (2002)67103.

[4] S. Kauffman, Metabolic stability and epigenesis in randomly connected nets, Journal of Theoretical Biology, 22 (1969) 437-467.

[5] S. Kauffman, Origins of Order Self-Organization and Selection in Evolution, Oxford University Press, 1993.

[6] L. Mendoza, D. Thieffry, E.R. Alvarez-Buylla, Flower's Morphogenesis of Arabidopsis ThalianaFlower's Morphogenesis of Arabidopsis Thaliana: a logical analysis, Bioinformatics, 15 (1999) 593-606.

[7] R. Thomas, Boolean formalization of genetic control circuits, Journal of Theoretical Biology, 42 (1973) 563-585.

[8] R. Thomas, R. d'Ari, Biological Feedback, CRC Press, 1990. 